\title{
ULTRAVIOLET, VISIBLE, AND INFRARED SPECTROSCOPY OF INTERSTELLAR
} MOLECULES

\author{
John H. Black \\ Steward Observatory \\ University of Arizona \\ Tucson, AZ 85721 USA
}

\begin{abstract}
Absorption line spectroscopy in the ultraviolet, visible, and infrared regions can provide important probes of interstellar chemistry. Significant recent developments include the application of optical absorption line techniques to the study of thick molecular clouds and the improvements in infrared detectors that will eventually lead to effective interstellar spectroscopy in the infrared. Demands for basic molecular data will grow in scope and in level of precision.
\end{abstract}

\section{INTRODUCTION}

Owing to improvements in detectors and in astronomical spectrographs, optical observations can now be applied to very thick interstellar clouds and to the investigation of finer details of the internal structure of diffuse clouds. As a result, the demand for basic spectroscopic data on simple molecules is expanding in scope and is becoming more severe with respect to precision. In some cases, exploratory work is needed to identify the electronic and vibrational spectra of important interstellar molecules like $\mathrm{C}_{3} \mathrm{H}$ and $\mathrm{C}_{3} \mathrm{H}_{2}$. In other instances, there are continuing needs for accurate oscillator strengths and for the resolution of discrepancies between experimental and theoretical determinations of them. Even in cases of well studied molecular spectra like those of $\mathrm{CH}$ and $\mathrm{CN}$, the measurement of line positions needs to be improved in accuracy by an order of magnitude or more to satisfy existing needs in the interpretation of astronomical observations.

The number of interstellar molecules detected by optical methods has not increased much since earlier reviews of the subject were completed (Snow 1980; Black 1985; Federman 1987). In the meantime, however, upper limits have been obtained for additional species and the variety of interstellar clouds investigated through known molecules has grown. A recent list of molecules sought by optical methods in diffuse clouds can be found in van Dishoeck and Black (1988a). In particular, no polyatomic molecule has been detected in the interstellar gas by optical absorption measurements. The impending launch of the Hubble Space Telescope (HST) promises more sensitive, new searches for molecules in the ultraviolet. Improvements in infrared spectroscopy should open up a new realm of molecular studies in the next few years. In this brief review, recent astronomical results that illustrate needs for molecular data are considered. Some recent developments in molecular studies relevant to astrochemistry are summarized. Finally, an attempt is made to anticipate some paths that the subject might profitably follow in the future. 


\section{BASIC SPECTROSCOPY}

Many of the molecules of astrochemical interest are radicals and ions that lack detailed spectroscopic analyses. Moreover, there exist some stable, unreactive species for which no high-resolution spectroscopy has been performed in the ultraviolet. Although most of the previously unidentified interstellar absorption lines in the ultraviolet spectrum of $\zeta$ Ophiuchi have since been assigned to $\mathrm{H}_{2}, \mathrm{HD}$, and atomic carbon, there remain as many as 19 unidentified interstellar lines at $\lambda=1023-1317 \AA$, some of which might be molecular (Morton 1978). We can expect that the number of unidentified ultraviolet lines will increase when the Hubble Space Telescope finally reaches orbit.

Interstellar absorption lines of $\mathrm{CH}$ were first identified more than 50 years ago (Swings and Rosenfeld 1937), yet increasing demands on the spectroscopy of $\mathrm{CH}$ continue to the present day. The widely observed interstellar $\mathrm{CH}$ feature $\mathrm{A}^{2} \Delta-\mathrm{X}^{2} \Pi(0,0) R_{f}(1 / 2)$ is a closely spaced $\Lambda$-doublet (wavelengths 4300.3030 and $4300.3235 \AA$ in standard air) which has never been resolved in astronomical spectra. With the recognition that some diffuse interstellar clouds exhibit complex Doppler velocity structure in multiple components on scales of $0.3-1.0 \mathrm{~km} \mathrm{~s}^{-1}$ (corresponding to wavelength shifts of $0.004-0.014 \AA$ at $4300 \AA$ ), the accurate description of the intrinsic line shape (i.e. doublet structure in this example) becomes essential to the interpretation of the observations (Black and van Dishoeck 1988a). Laboratory measurements adequate for this purpose have only recently become available (Brazier and Brown 1984; Bernath 1987). Furthermore, the mean wavelength of the 4300 $\AA$ line blend differs by $0.008 \AA$ (i.e. $0.54 \mathrm{~km} \mathrm{~s}^{-1}$ in Doppler velocity) from the value previously adopted in the astronomical literature. The wavelengths of the interstellar lines of the $\mathrm{B}^{2} \Sigma^{-}-\mathrm{X}^{2} \Pi(0,0)$ band of $\mathrm{CH}$ have also been determined much more accurately $(\mathrm{P}$. F. Bernath, preliminary results, as quoted by Black and van Dishoeck 1988a).

Interstellar absorption lines of CN are used not only to study its abundance and chemistry, but also as an indirect radiometric sensor of the cosmic background radiation, which governs the rotational excitation of $\mathrm{CN}$ in interstellar clouds of low density. In denser regions, the measurement of excess excitation can be used to estimate the electron density. The most recent observations of CN in diffuse clouds (Meyer and Jura 1985; Crane et al. 1986) determine the relative populations in the $N=0$ and $N=1$ rotational levels (or the excitation temperature) to approximately $\pm 1 \%$ accuracy. At this level, the extraction of the true values of the column densities and the excitation temperature from the measurements can be quite sensitive to very small amounts of line saturation. The unresolved spin-doublets of the $\mathrm{CN} \mathrm{B}^{2} \Sigma^{+}-\mathrm{X}^{2} \Sigma^{+}$(violet) system have traditionally been treated as single, unresolvable lines. In fact, the B-X $(0,0) R(0)$ feature is a blend of $R_{1}(0)$ and ${ }^{R} Q_{21}(0)$ lines with respective relative strengths of $2 / 3$ and $1 / 3$ and with a separation estimated to be $0.0035 \mathrm{~A}\left(0.27 \mathrm{~km} \mathrm{~s}^{-1}\right.$ in Doppler velocity) on the basis of published spectroscopic constants (Engleman 1974). This small splitting is uncertain and needs to be determined more accurately. There exist observed interstellar CN lines (Meyer and Hawkins 1987) for which the neglect of the unresolved structure would cause a $40 \%$ overestimate of the ground-state column density if the Doppler line broadening parameter were as small as $b=0.5 \mathrm{~km} \mathrm{~s}^{-1}$ (van Dishoeck and Black 1989).

Spectroscopic studies of small, carbon-bearing molecules of astrophysical interest have produced interesting results recently. The $\mathrm{B}^{4} \Sigma_{u}^{-}-\mathrm{X}^{4} \Sigma_{g}^{-}$electronic transition of $\mathrm{C}_{2}^{+}$has been identified ( $O$ 'Keefe et al. 1984; Forney et al. 1987) and subsequently given a rotational anaylsis (Rösslein et al. 1987). The $(0,0)$ band is calculated to have an oscillator strength $f_{00}=0.0156$ (Rosmus et al. 1986), and potential interstellar absorption lines are at convenient wavelengths: $(0,0) R(1), 5065.19 \AA ; R(3), 5063.29 \AA$. However, $\mathrm{C}_{2}^{+}$reacts rapidly with $\mathrm{H}_{2}$; therefore, its interstellar abundance is predicted to be quite low. Abundances of 
the related ions $\mathrm{C}_{2} \mathrm{H}^{+}$and $\mathrm{C}_{2} \mathrm{H}_{2}^{+}$are likely to be higher and similar spectroscopic analyses of them would be valuable. Photoelectron spectroscopy of $\mathrm{C}_{2} \mathrm{H}_{2}^{+}$indicates that the origin of the $\tilde{\mathrm{A}}^{2} A_{g}-\tilde{\mathrm{X}}^{2} \Pi_{u}$ system should lie near $\lambda=2533 \AA$ (Reutt et al. 1986). Moreover, the $\nu_{3}$ fundamental band of this ion has been observed directly at $\tilde{\nu}=3136 \mathrm{~cm}^{-1}$ (Crofton et al. 1987). Further exploratory spectroscopy is also needed for the ion $\mathrm{C}_{2} \mathrm{~N}^{+}$, whose interstellar abundance may be high (Hartquist and Dalgarno 1980) and whose rotational spectrum has been investigated through theoretical calculations (Kraemer et al. 1984; Jensen and Kraemer 1988). The electronic spectrum of the neutral CCN is fairly well known (see Brazier et al. 1987 and references therein). It would also be of great interest to know about the electronic transitions of the first interstellar ring molecule, cyclopropenylidene $\left(\mathrm{C}_{3} \mathrm{H}_{2}\right)$, because it is observed to be widespread and abundant even in diffuse clouds (Cox et al. 1988) through its pure rotational transitions. Electronic and vibrational spectra of $\mathrm{C}_{3} \mathrm{H}$, which has been observed in both cyclic and linear forms, would be of interest.

The $\mathrm{C}_{3}$ molecule is observed in comets and carbon stars and has been sought unsuccessfully in interstellar clouds through its $\tilde{\mathrm{A}}^{1} \Pi_{u}-\tilde{\mathrm{X}}^{1} \Sigma_{g}^{+}$transition at $4050 \AA$ (Clegg and Lambert 1982; Snow et al. 1988). $C_{3}$ is expected to have an ultraviolet transition ${ }^{1} \Sigma_{u}^{+}-\tilde{X}^{1} \Sigma_{g}^{+}$with a very large oscillator strength, $f \approx 0.9$ (Römelt et al. 1978). Although ultraviolet absorption at $1580 \AA$ has been attributed to $C_{3}$ (Shinn 1982) and matrix isolation studies indicate that the $(0,0,0)-(0,0,0)$ band occurs near $1893 \AA$ (Chang and Graham 1982 ), a rotational analysis based on a gas-phase spectrum is still needed. More recently, the $\nu_{3}$ fundamental vibration-rotation band of $\mathrm{C}_{3}$ has been identified and analyzed in the spectrum of the carbon star IRC+10216 (Hinkle et al. 1988) and in laboratory spectra (Matsumura et al. 1988). The oscillator strength of this band is large for an infrared transition (Kraemer et al. 1984), and interstellar $C_{3}$ is potentially observable in thick clouds through absorption lines near $4.9 \mu \mathrm{m}$ wavelength. Positions of the interstellar absorption lines of the Phillips system of $\mathrm{C}_{2}$ can now be determined much more precisely as a result of the work of Douay et al. (1988). The $\mathrm{C}_{2} \mathrm{H}$ molecule, which is widely observed through its rotational transitions at millimeter wavelengths, has an electronic transition in the infrared (Curl et al. 1985) for which transition moments have been calculated (Reimers et al. 1985).

As high-resolution infrared spectrometers with more sensitive array detectors come into operation, infrared spectroscopy of interstellar molecules will begin to play a significant role in astrochemistry. The most abundant interstellar molecule, $\mathrm{H}_{2}$, has been essentially unobservable in thick, quiescent molecular clouds, although it can be observed by means of ultraviolet absorption lines in thin, diffuse clouds and through infrared emission lines from molecular gas that is highly disturbed by shock waves or exposed to intense ultraviolet radiation. The fact that its infrared lines are weak dipole-forbidden, electric quadrupole transitions would seem to preclude absorption line studies; however, column densities of $\mathrm{H}_{2}$ of the order of $10^{23} \mathrm{~cm}^{-2}$ or less are in principle detectable in absorption in the $(1,0) S(0)$ and $S(1)$ vibration-rotation lines toward suitably located infrared sources. In this instance, "detectable" refers to an equivalent width $W_{\nu} \leq 0.01 \mathrm{~cm}^{-1}$ at $\lambda=2.1-2.2 \mu \mathrm{m}$, which requires only a signal/noise ratio of 10 or more at a resolving power $\lambda / \Delta \lambda \approx 10^{5}$. Moreover, it should be possible to observe infrared absorption lines of $\mathrm{CO}$ and $\mathrm{H}_{2}$ simultaneously and thus to make direct determinations of the $\mathrm{CO} / \mathrm{H}_{2}$ abundance ratio, which is important for quantitative studies of the distribution of molecular material and the efficiency of star formation in galaxies (Williams 1985; van Dishoeck and Black 1987). The infrared absorption lines of interstellar $\mathrm{CO}$ have already been detected, both in actively star-forming regions (Hall et al. 1978; Scoville et al. 1983; Geballe and Wade 1985) and in a quiescent cloud (Black and Willner 1984). Solid CO that has frozen onto interstellar grains has been identified through its distinctive infrared absorption spectrum (Hagen et al. 1980; Lacy et al. 1984; Larson et al. 1985; Whittet et al. 1985; Geballe et al. 1985; Geballe 1986). Recent 
laboratory investigations have also made it possible to determine the column density of solid $\mathrm{CO}$ and to infer something about the surface chemistry (Sandford et al. 1988). Laboratory spectroscopy of other astrophysical ices has also been pursued (e.g. d'Hendecourt and Allamandola 1986, and references therein).

Infrared absorption line spectroscopy can provide significant tests of theories of interstellar chemistry, especially with respect to the abundances of important non-polar species like $\mathrm{H}_{3}^{+}, \mathrm{CH}_{4}$, and $\mathrm{C}_{2} \mathrm{H}_{2}$, which are otherwise not readily observable in interstellar space. The infrared absorption lines of $\mathrm{H}_{3}^{+}$at $\lambda=3.7-4.1 \mu \mathrm{m}$ are expected to be detectable both in dark molecular clouds (Oka 1981) and in classical diffuse clouds (van Dishoeck and Black 1986; Black and van Dishoeck 1988b). Knacke et al. (1985) have reported a recent search for interstellar $\mathrm{CH}_{4}$. Table 1 lists a selection of infrared transitions of actual or potential interstellar molecules that will be important for absorption line studies. The table presents band or line frequencies in $\mathrm{cm}^{-1}$ and, where information on oscillator strengths is available, a characteristic column density required to produce an absorption feature of equivalent width $W_{\nu}=0.01 \mathrm{~cm}^{-1}$.

Metal atoms and ions may play a major role in the ionization balance of interstellar clouds, but very little is known about their participation in interstellar chemistry. Many metal-containing molecules have strong, well known transitions in the visible region. However, no molecules containing $\mathrm{Mg}, \mathrm{Ca}, \mathrm{Na}, \mathrm{Fe}$, or $\mathrm{Ti}$ have been found in the interstellar medium, with the possible exception of a detection of $\mathrm{MgO}$ at millimeter wavelengths. No sensitive optical searches for molecules like $\mathrm{MgO}$ and $\mathrm{TiO}$ have ever been reported in the literature. In view of the possibility that $\mathrm{TiO}^{+}$forms by an exothermic chemi-ionization process in interstellar clouds, $\mathrm{Ti}+\mathrm{O} \rightarrow \mathrm{TiO}^{+}+\mathrm{e}$, it would be interesting to know something about the spectrum of this ion (Oppenheimer and Dalgarno 1977, Black 1988).

The subject of very large molecules (e.g. polycyclic aromatic hydrocarbons = PAHs) in interstellar space is very fashionable (see, e.g., Omont 1986, Lepp and Dalgarno 1988, and references therein) and much spectroscopic work remains to be done. Although PAHs have joined the long list of possible carriers of the diffuse interstellar bands (Josafatsson and Snow 1987 and references therein), no specific and convincing identification has been made. The large molecule buckminsterfullerene, $\mathrm{C}_{60}$, has recently been shown to have an isolated, narrow absorption band at $3860 \AA$ (Heath, Curl, and Smalley 1987).

\section{QUANTITATIVE SPECTROSCOPY}

Molecular abundances can be determined and quantitative tests of chemical models can be made only if oscillator strengths of molecular transitions are known. Only a few recent developments can be summarized here; for a recent review, see Smith (1987). Although detections or upper limits have been reported for at least 34 molecules in diffuse clouds (see Table 1 of van Dishoeck and Black 1988a), several of the limits are not very useful owing to the lack of information on oscillator strengths. Specifically, oscillator strengths are needed for ultraviolet transitions of $\mathrm{MgH}^{+}, \mathrm{SH}, \mathrm{CaH}$, and $\mathrm{SiO}$. Naturally, oscillator strengths will also be needed for various species whose electronic spectra have not yet been identified and analyzed (see $\$ 2$ above).

The red systems of $\mathrm{CN} \mathrm{A}^{2} \Pi-\mathrm{X}^{2} \Sigma^{+}$and $\mathrm{C}_{2} \mathrm{~A}^{1} \Pi_{u}-\mathrm{X}^{1} \Sigma_{g}^{+}$are now being used for absorption line studies of diffuse and translucent clouds (van Dishoeck and Black 1989). Although much effort has been devoted to the determination of the oscillator strengths of these systems, there remain some unresolved questions. Recent experiments are in harmony for the $\mathrm{C}_{2}$ A-X Phillips system (Erman et al. 1982; Bauer et al. 1985, 1986; Davis et al. 1984) with $f_{00} \approx 1.4 \times 10^{-3}$ and $f_{20} \approx 1.0 \times 10^{-3}$; however, oscillator strengths determined 
Table 1. SELECTED INFRARED TRANSITIONS OF INTERSTELLAR MOLECULES

\begin{tabular}{|c|c|c|c|}
\hline Species & Transition & $\tilde{\nu}\left(\mathrm{cm}^{-1}\right)$ & $N_{0}\left(\mathrm{~cm}^{-2}\right)$ \\
\hline $\mathbf{H}_{\mathbf{2}}$ & $v=1-0 \mathrm{~S}(0)$ & 4497.8391 & $1.2(23)$ \\
\hline $\mathrm{H}_{8}^{+}$ & $\nu_{2}=1-0{ }^{\dot{R}} \dot{R}_{1}(1)$ & 2691.444 & $1.3(14)$ \\
\hline $\mathrm{H}_{2} \mathrm{D}^{+}$ & $\begin{array}{l}\nu_{1}=1-01_{01}-0_{00} \\
\nu_{3}=1-0\end{array}$ & $\begin{array}{l}3038.177 \\
2329 .\end{array}$ & $\begin{array}{l}2.3(15) \\
4.9(14)\end{array}$ \\
\hline $\mathrm{HeH}^{+}$ & $v=1-0 R(0)$ & 2972.91 & $7.6(13)$ \\
\hline $\begin{array}{l}\mathrm{H}^{85} \mathrm{Cl} \\
\mathrm{CO}\end{array}$ & $\begin{array}{l}v=1-0 \mathrm{R}(0) \\
v=1-0 \mathrm{R}(0) \\
v=2-0 \mathrm{R}(0)\end{array}$ & $\begin{array}{l}2906.2474 \\
2147.081 \\
\mathbf{4 2 6 3 . 8 9 7 3 4}\end{array}$ & $\begin{array}{l}1.8(15) \\
1.1(15) \\
1.5(17)\end{array}$ \\
\hline $\mathrm{CN}$ & $\begin{array}{l}\mathrm{A}^{2} \Pi-\mathrm{X}^{2} \Sigma^{+}(0,0) \mathrm{R}_{1}(0) \\
v=1-0 \\
v=2-0\end{array}$ & $\begin{array}{l}9094.3192 \\
2046.13 \\
4062.26\end{array}$ & $\begin{array}{l}6.6(12) \\
2.6(15) \\
8.0(15)\end{array}$ \\
\hline $\begin{array}{l}\mathrm{CH}^{+} \\
\mathrm{CH}^{-} \\
\mathrm{CH}_{2}\end{array}$ & $\begin{array}{l}v=1-0 \mathrm{R}(0) \\
v=1-0 \mathrm{R}_{2}(1 / 2) \\
\nu_{1}=1-0 \\
\nu_{3}=1-0\end{array}$ & $\begin{array}{l}2766.5341 \\
2796.885,2797.008 \\
3020.7 \\
\mathbf{3 1 5 2 . 5}\end{array}$ & $\begin{array}{l}3.4(16) \\
6.0(14)\end{array}$ \\
\hline $\begin{array}{l}\mathrm{CH}_{3} \\
\mathrm{CH}_{4} \\
\mathrm{HC}_{3} \mathrm{~N}\end{array}$ & $\begin{array}{l}\nu_{3}=1-01_{1}-0_{0} \\
\nu_{3}=1-0 \\
\nu_{1}=1-0 R(0)\end{array}$ & $\begin{array}{l}\mathbf{3 1 7 4 . 2 9 3 5} \\
\mathbf{3 0 1 9} \\
\mathbf{3 3 2 7 . 6 8 0}\end{array}$ & $\begin{array}{l}6.0(15) \\
9.4(14)\end{array}$ \\
\hline \multirow[t]{3}{*}{$\mathrm{C}_{2}$} & $\mathrm{~A}^{1} \Pi_{u}-\mathrm{X}^{1} \Sigma_{g}^{+}(0,0) \mathrm{Q}(2)$ & 8267.1336 & $1.6(13)$ \\
\hline & $\mathrm{A}^{1} \mathrm{I}_{u}-\mathrm{X}^{1} \Sigma_{o}^{+}(1,0) \mathrm{Q}(2)$ & 9851.1169 & $1.4(13)$ \\
\hline & $A^{1} \Pi_{u}-X^{1} \Sigma_{g}^{+}(2,0) Q(2)$ & 11410.84115 & $2.3(13)$ \\
\hline $\mathbf{C}_{3}$ & $\nu_{3}=1-0$ & 2042.665 & $9.7(13)$ \\
\hline \multirow[t]{4}{*}{$\mathrm{C}_{2} \mathrm{H}_{2}$} & $\begin{array}{l}\tilde{A}^{2} \Pi(0,0,0)-\hat{X}^{2} \Sigma^{+}(0,0,0) \\
010(11)_{+}^{0} R(1)\end{array}$ & $\begin{array}{l}3772-4108 \\
3286.585\end{array}$ & $\begin{array}{l}1.0(14) \\
2.4(15)\end{array}$ \\
\hline & $0010^{\circ} 0^{\circ} \mathrm{R}(1)$ & 3299.521 & $2.9(15)$ \\
\hline & $1000^{\circ} 1^{1} Q(1)$ & 4091.170 & $6.2(16)$ \\
\hline & $0100^{0} \mathrm{~g}^{1} \mathrm{Q}(1)$ & 4138.888 & \\
\hline $\begin{array}{l}\mathrm{HCO}+ \\
\mathrm{HCN} \\
\text { DCN }\end{array}$ & $\begin{array}{l}\nu_{1}=1-0 \mathbf{R}(0) \\
\nu_{3}=1-0 \\
\nu_{2}=1-0\end{array}$ & $\begin{array}{l}3091.679 \\
3311 .\end{array}$ & $\left.\begin{array}{l}1.0 \\
1.8 \\
15\end{array}\right)$ \\
\hline $\begin{array}{l}\text { DCN } \\
\mathrm{C}^{82} \mathrm{~S}\end{array}$ & $\begin{array}{l}\nu_{s}=1-0 \\
v=1-0 \mathrm{R}(0)\end{array}$ & $\begin{array}{l}2630 . \\
1273.7898\end{array}$ & $1.8(15)$ \\
\hline $\begin{array}{l}\mathrm{OH}^{2} \\
\mathrm{NH}_{2}\end{array}$ & $\begin{array}{l}v=1-0 Q_{1}(3 / 2) \\
\nu_{1}=1-01_{11}-0_{00}\end{array}$ & $\begin{array}{l}3568.47 \\
3250.54\end{array}$ & $9.1(15)$ \\
\hline $\begin{array}{l}\text { SHF } \\
\text { HF }\end{array}$ & $\begin{array}{l}\nu_{8}=1-01_{01}-000 \\
v=1-0 \\
v=1-0 \mathrm{R}(0)\end{array}$ & $\begin{array}{l}3322.50 \\
2598.08 \\
4000.9894\end{array}$ & $6.4(14)$ \\
\hline
\end{tabular}

Note: The characteristic column density, $N_{0}$, is that required to produce a feature with an equivalent width of $0.01 \mathrm{~cm}^{-1}$ in the weak-line limit. Each transition is designated either as a band or as an individual line. In the former case, the characteristic column density refers to a sum over all lower rotational levels; in the latter case, the column density is that in the lower level of the line.

from $a b$ initio theoretical calculations remain factors of 1.5 - 2 larger (van Dishoeck 1983; Chabalowski et al. 1983; Pouilly et al. 1983; ONeil et al. 1987; Klotz 1987). Theory and 
experiment are similarly discrepant for the A-X system of CN (Cartwright and Hay 1982; Larsson et al. 1983; Davis et al. 1986; Taherian and Slanger 1984; Sneden and Lambert 1982), although very recent calculations (Bauschlicher et al. 1988) come closer to experiment. Since the oscillator strength of the B-X system is well established, consistency tests can be carried out by astronomical spectroscopy for a few regions where both the violet and red system lines of interstellar CN are observed.

There remains an unresolved disagreement between two lifetime measurements for the $\tilde{\mathrm{A}}-\tilde{\mathrm{X}}$ system of $\mathrm{H}_{2} \mathrm{O}^{+}$(Möhlmann et al. 1978; Curtis and Erman 1977), which is significant in view of the very low limits that have been placed on the strengths of its interstellar absorption lines in several clouds (see Federman 1987). This is important for the study of cometary spectra as well (see Lutz 1987).

In the Lyman and Werner systems in the ultraviolet spectrum of $\mathrm{H}_{2}$, non-adiabatic rotational perturbations affect the oscillator strengths of individual rotational lines: the calculated line strengths of Ford (1975) have recently been extended to a larger number of transitions by Abgrall et al. (1987). Accurate transition probabilities in the $\nu_{2}$ vibrationrotation band of $\mathrm{H}_{3}^{+}$have been calculated by Miller and Tennyson (1988).

Another important aspect of quantitative spectroscopy concerns information that is required for the full description of processes like photodissociation. One example of much current interest is the photodissociation of $\mathrm{CO}$, which evidently occurs principally through pre-dissociating lines rather than by continuous absorption in the interstellar medium. Information on line positions, isotopic shifts, oscillator strengths, and pre-dissociation widths is becoming available as a result of diligent efforts by Eidelsberg et al. (1984), Letzelter et al. (1987), Stark et al. (1987), and Yoshino et al. (1988). The new spectroscopic data have already been applied to theoretical models of the CO chemistry in circumstellar envelopes (Mamon et al. 1988) and in interstellar clouds (Viala et al. 1988; van Dishoeck and Black 1988b). The rate of photodissociation of CO depends upon the intensity of ultraviolet starlight and on the oscillator strengths of the dissociating lines, while the depth-dependence of the absorption is governed partly by the line broadening which has a signicant contribution from the pre-dissociation itself. Observationally, the translucent clouds may provide the most important tests of our understanding of the CO photochemistry in that they occupy the realm of parameter space in which $\mathrm{CO}$ is just beginning to account for a large fraction of the gas-phase carbon and oxygen. Ultraviolet absorption line observations with the High Resolution Spectrograph on HST promise to be of some value in this regard since there are many strong bands of the $\mathrm{A}^{1} \Pi^{-}-\mathrm{X}^{1} \Sigma^{+}$ 4th-positive system at wavelengths as long as $1544 \AA$. On the other hand, these absorption features will be strongly saturated in translucent clouds, and the interpretation will be quite complicated owing to the dense rotational structure of these bands, which will at best be barely resolved at the highest resolution of the High Resolution Spectrograph. The fundamental and first overtone vibration-rotation bands of $\mathrm{CO}$ at 4.6 and $2.3 \mu \mathrm{m}$ will eventually provide an important alternative source of information on the abundance and excitation of this molecule. Although atomic spectroscopy is outside the purview of this review, the chemistry of simple carbon-bearing molecules cannot be fully understood without knowledge of the abundances of $\mathrm{C}$ and $\mathrm{C}^{+}$. HST is expected to make possible sensitive interstellar absorption measurements of the $\mathrm{C}$ II] intersystem multiplet near 2325 $\AA$; therefore, it is important to determine the small oscillator strengths of these lines as accurately as possible (Nussbaumer et al. 1981; Stencel et al. 1981; Hobbs et al. 1982; Cowan et al. 1982; Lennon et al. 1985). 
Preparation of this review has been supported in part by NASA through grant NAGW-763 to the University of Arizona. E. F. van Dishoeck provided very helpful comments.

\section{REFERENCES}

Abgrall, H., Launay, F., Roueff, E., and Roncin, J.-Y. 1987, J. Chem. Phys., 87, 2086.

Bauer, W., Becker, K. H., Hubrich, C., Meuser, R., and Wildt, J. 1985, Ap. J., 296, 758.

Bauer, W., Becker, K. H., Bielefeld, M., and Meuser, R. 1986, Chem. Phys. Lett., $123,35$.

Bauschlicher, C. W., Langhoff, S. R., and Taylor, P. R. 1988, Ap. J., 932, 531

Bernath, P. F. 1987, J. Chem. Phys., 86, 4888.

Black, J. H. 1985, in Molecular Astrophysics: State of the Art and Future Directions, G. W. F. Diercksen, W. F. Huebner, and P. W. Langhoff, editors, (Dordrecht: Reidel), p. 215.

Black, J. H. 1988, Adv. Atom. Mol. Phys., 25, in press.

Black, J. H., and van Dishoeck, E. F. 1988a, Ap. J., S31, 986.

Black, J. H., and van Dishoeck, E. F. 1988b, to appear in Dissociative Recombination, J. B. A. Mitchell and S. Guberman, editors, (Singapore: World Scientific), in press.

Black, J. H., and Willner, S. P. 1984, Ap. J., 279, 673.

Brasier, C. R., and Brown, J. M. 1984, Canadian J. Phys., 62, 1563.

Brasier, C. R., O'Brien, L. C., and Bernath, P. F. 1987, J. Chem. Phys., 86, 3078.

Cartwright, D. C., and Hay, P. J. 1982, Ap. J., $257,383$.

Chabalowaki, C. F., Peyerimhoff, S. D., and Buenker, R. J. 1983, Chem. Phys., $81,57$.

Chang, K. W., and Graham, W. R. M. 1982, J. Chem. Phys., 77, 4300.

Clegg, R. E. S., and Lambert, D. L. 1982, M. N. R. A. S., 201, 728.

Cox, P., Güsten, R., and Henkel, C. 1988, Astr. Ap., in press.

Cowan, R. D., Hobbs, L. M., and York, D. G. 1982, Ap. J., 257, 373; 265, 582.

Crane, P., Hegyi, D. J., Mandolesi, N., and Danks, A. C. 1986, Ap. J., s09, 822.

Crofton, M. W., Jagod, M.-F., Rehfuss, B. D., and Oka, T. 1987, J. Chem. Phys., 86, 3755.

Curl, R. F., Carrick, P. G., and Merer, A. J. 1985 J. Chem. Phys., 82, 3479.

Curtis, L. J., and Erman, P. 1977, J. Opt. Soc. Am., 67, 1218.

Davis, S. P., Smith, W. H., Brault, J. W., Pecyner, R., and Wagner, J. 1984, Ap. J., $287,455$.

Davis, S. P., Shortenhaus, D., Stark, G., Engleman, R., Phillips, J. G., and Hubbard, R. P. 1986, Ap. J., 303, 892.

d'Hendecourt, L. B., and Allamandola, L. J. 1986, Astr. Ap. Suppl., 64, 453.

Douay, M., Nietmann, R., and Bernath, P. F. 1988, J. Mol. Spectrosc., in press.

Eidelsberg, M., Lannay, F., Rostas, F., Le Floch, A., Breton, J., and Thieblemont, B. 1984, Ann. Isr. Phys. Soc. (Israel), 6, 240.

Engleman, R. 1974, J. Mol. Spectrosc., 49, 106.

Erman, P., Lambert, D. L., Larwson, M., and Mannfors, B. 1982, Ap. J., 25s, 983.

Federman, S. R. 1987, in IAU Symposium 120, Astrochemistry, eds. M.S. Vardya and S.P. Tarafdar (Reidel, Dordrecht), p. 12s.

Ford, A. L. 1975, J. Mol. Spectrosc., 56, 251.

Forney, D., Althaus, H., and Maier, J. P. 1987, J. Phys. Chem., 91, 6458.

Geballe, T. R. 1986, Astr. Ap., 162, 248.

Geballe, T. R., Bass, F., Greenberg, J. M., and Schutte, W. 1985, Astr. Ap., 146, L6.

Geballe, T. R., and Wade, R. 1985, Ap. J.'(Letters), 291, L55.

Hagen, W., Allamandola, L. J., and Greenberg, J. M. 1980, Astr. Ap., 86, Ls.

Hall, D. N.' B., Kleinmann, S. G., Ridgway, S. T., and Gillett, F. C. 1978, Ap. J. (Letters), 228, L47.

Hartquist, T. W., and Dalgarno, A. 1980, in Giant Molecular Clouds in the Galaxy, P. M. Solomon and M. G. Edmunds, editors, (Oxford: Pergamon), p. 315.

Heath, J. R., Curl, R. F., and Smalley, R. E. 1987, J. Chem. Phys., 87, 4236.

Hinkle, K. H., Keady, J. J., and Bernath, P. F. 1988, Science, 241, 1319.

Hobbs, L. M., York, D. G., and Oegerle, W. 1982, Ap. J. (Letters), 252, L21.

Jensen, P., and Kraemer, W. P. 1988, J. Mol. Spectrosc., 129, 216.

Josafatsson, K., and Snow, T. P. 1987, Ap. J., \$19, 436.

Klots, R. 1987, private communication.

Knacke, R. F., Geballe, T. R., Noll, K. S., and Tokunaga, A. T. 1985, Ap. J. (Letters), 298, L67.

Kraemer, W. P., Bunker, P. R., and Yoshimine, M. 1984, J. Mol. Spectrasc., 107, 191. 
Lacy, J. H., Baas, F., Allamandola, L. J., Persson, S. E., McGregor, P. J., Lonsdale, C. J., Geballe, T. R., and van de Bult, C. E. P. 1984, Ap. J., 276, 533.

Larson, H. P., Davis, D. S., Black, J. H., and Fink, U. 1985, Ap. J., $299,873$.

Larsson, M., Siegbahn, P. E. M., and Ågren, H. 1983, Ap. J., 272, 369.

Lennon, D. J., Dufton, P. L., Hibbert, A., and Kingston, A. E. 1985, Ap. J., 294, 200.

Lepp, S., and Dalgarno, A. 1988 Ap. J., 324, 553.

Letzelter, C., Edielsberg, M., Rostas, F., Breton, J., and Thieblemont, B. 1987, Chem. Phys., 114, 273.

Lutz, B. L. 1987, Ap. J. (Letters), 315, L147.

Mamon, G. A., Glassgold, A. E., and Huggins, P. J. 1988, Ap. J., 328, 797.

Matsumura, K., Kanamori, H., Kawaguchi, K., and Hirota, E. 1988, J. Chem. Phys., 89, 3491.

Meyer, D. M., and Hawkins, I. 1987, Bull. Am. Astr. Soc., 19, 1054.

Meyer, D. M., and Jura, M. 1985, Ap. J., $297,119$.

Miller, S., and Tennyson, J. 1988, Ap. J., in press.

Möhlmann, G. R., Bhutani, K. K., de Heer, F. J., and Tsurubuchi, S. 1978, Chem. Phys., 31, 273.

Morton, D. C. 1978, M. N. R. A. S., 184, 713.

Nussbaumer, H., and Storey, P. J. 1981, Astr. Ap., 96, 91.

Oka, T. 1981, Phil. Trans. R. Soc. London A, 303, 543.

O'Keefe, A., Derai, R., and Bowers, M. T. 1984 Chem. Phys., 91, 161.

Omont, A. 1986, Astr. Ap., 164, 159.

ONeil, S. V., Rosmus, P., and Werner, H.-J. 1987, J. Chem. Phys., 87, 2847.

Oppenheimer, M., and Dalgarno, A. 1977, Ap. J., $212,683$.

Pouilly, B., Robbe, J. M., Schamps, J., and Roueff, E. 1983, J. Phys. B, 16, 437.

Reimers, J. R., Wilson, K. R., Heller, E. J., and Langhoff, S. R. 1985, J. Chem. Phys., 82, 5064.

Reutt, J. E., Wang, L. S., Pollard, J. E., Trevor, D. J., Lee, Y. T., and Shirley, D. A. 1986, J. Chem. Phys., 84, 3022.

Römelt, J., Peyerimhoff, S. D., and Buenker, R. J. 1978, Chem. Phys. Lett., 58, 1.

Rosmus, P., Werner, H.-J., Reinsch, E.-A., and Larsson, M. 1986, J. Elec. Spectrosc. Related Phen., 41, 289.

Rösslein, M., Wyttenbach, M., and Maier, J. P. 1987, J. Chem. Phys., 87, 6770.

Sandford, S. A., Allamandola, L. J., Tielens, A. G. G. M., and Valero, G. J. 1988, Ap. J., 329, 498.

Scoville, N. Z., Kleinmann, S. G Hall, D. N. B., and Ridgway, S. T. 1983, Ap. J., $275,201$.

Shinn, J. L. 1982, in "Thermoprysics of Atmospheric Entry", T. E. Horton, editor, Prog. Astronaut. Aeronaut., 82, 68.

Smith, P. L. 1987, in IAU Symposium 120, Astrochemistry, eds. M.S. Vardya and S.P. Tarafdar (Reidel, Dordrecht), p. 95.

Sneden, C., and Lambert, D. L. 1982, Ap. J., $259,381$.

Snow, T. P. 1980, in IAU Symposium 87, Interstellar Molecules, ed. B.H. Andrew (Reidel, Dordrecht), p. 247.

Snow, T. P., Seab, C. G., and Joseph, C. L. 1988, Ap. J., submitted.

Stark, G., Smith, P. L., Yoshino, K., and Parkinson, W. H. 1987, private communication, and in preparation.

Stencel, R. E., Linsky, J. L., Brown, A., Jordan, C., Carpenter, K. G., Wing, R. F., and Czyzak, S. 1981, M. N.R. A. S., 196, 4P.

Swings, P., and Rosenfeld, L. 1937, AP. J., 86, 483.

Taherian, M. R., and Slanger, T. G. 1984, J. Chem. Phys., 81, 3814.

van Dishoeck, E. F. 1983, Chem. Phys., 77, 277.

van Dishoeck, E. F., and Black, J. H. 1986, Ap. J. Suppl., 62, 109.

van Dishoeck, E. F., and Black, J. H. 1987, in Physical Processes in Interstellar Clouds, eds. G. Morfill and M.S. Scholer (Reidel, Dordrecht), p. 241.

van Dishoeck, E. F., and Black, J. H. 1988a, in Rate Coefficients in Astrochemistry, T. J. Millar and D. A. Williams, editors, (Dordrecht: Kluwer), p. 209.

van Dishoeck, E. F., and Black, J. H. 1988b, Ap. J., 334, in press.

van Dishoeck, E. F., and Black, J. H. 1989, Ap. J., S40, in press.

Viala, Y. P., Letzelter, C., Eidelsberg, M., and Rostas, M. 1988, Astr. Ap., 198, 265.

Whittet, D. C. B., Longmore, A. J., and McFadzean, A. D. 1985, M. N. R. A. S., 216, 45P.

Williams, D. A. 1985, Quart. J. R. Astr. Soc., $26,463$.

Yoshino, K., Stark, G., Smith, P. L., Parkinson, W. H., and Ito, K. 1988, J. de Phys., in press. 